\title{
Algebrability of the set of non-convergent Fourier series
}

\author{
by \\ Richard M. Aron (Kent, OH), David PÉrez-García (Madrid) \\ and Juan B. Seoane-Sepúlveda (Kent, OH)
}

\begin{abstract}
We show that, given a set $E \subset \mathbb{T}$ of measure zero, the set of continuous functions whose Fourier series expansion is divergent at any point $t \in E$ is dense-algebrable, i.e. there exists an infinite-dimensional, infinitely generated dense subalgebra of $\mathcal{C}(\mathbb{T})$ every non-zero element of which has a Fourier series expansion divergent in $E$.
\end{abstract}

1. Introduction and preliminaries. Many examples of functions with some special or pathological properties have been found in analysis. Examples such as continuous nowhere differentiable functions, everywhere surjective functions, or differentiable nowhere monotone functions have been constructed in the past. Given such a special property, we say that the subset $M$ of functions which satisfy it is spaceable if $M \cup\{0\}$ contains a closed infinite-dimensional subspace. The set $M$ will be called (dense) lineable if $M \cup\{0\}$ contains an infinite-dimensional (dense) vector space. At times, we will be more specific, referring to the set $M$ as $\mu$-lineable if it contains a vector space of dimension $\mu$. Also, we let $\lambda(M)$ be the maximum cardinality (if it exists) of such a vector space. We believe that the terms lineable and spaceable were first introduced in [5] and, later, in [2] and [7] (see also [1]).

Some of these special properties are not isolated phenomena. In [2] it was shown that the set of everywhere surjective functions is $2^{\text {card( }(\mathbb{R})}$-lineable and that the set of differentiable functions on $\mathbb{R}$ which are nowhere monotone is lineable in $\mathcal{C}(\mathbb{R})$. Fonf, Gurariy and Kadets showed ([6]) that the set of nowhere differentiable functions on $[0,1]$ is spaceable in $\mathcal{C}[0,1]$. Some of these pathological behaviors occur in really interesting ways. For instance, in [11] Rodríguez-Piazza proved that every separable Banach space is isometric to a space of continuous nowhere differentiable functions, and in [8], Hencl showed that any separable Banach space is isometrically isomorphic

2000 Mathematics Subject Classification: Primary 42A20, 46E25; Secondary 42A16.

Key words and phrases: Fourier series, divergent series, lineability, spaceability, algebrability.

Partially supported by MTM2005-00082. 
to a subspace of $\mathcal{C}[0,1]$ whose non-zero elements are nowhere approximately differentiable and nowhere Hölder.

This paper continues with the search for sets of functions enjoying special properties, in particular, continuous functions whose Fourier series expansion diverges on a set of Lebesgue measure zero.

The convergence of Fourier series has been deeply studied in the past. It came as a considerable surprise when Du Bois-Reymond produced an example of a continuous function $f: \mathbb{T} \rightarrow \mathbb{C}$ whose Fourier series is divergent at one point (see [10, pp. 67-73] for a modern reference). This result can be improved by means of an example of a continuous function whose Fourier series expansion diverges on a set of measure zero $([9$, p. 58]). This last result is the best possible, since the Fourier expansion of every continuous function converges almost everywhere (Carleson, see e.g. [10, p. 75]). Moreover, by means of Baire's theorem, the pathological behavior can be shown to be generic: there exists a $G_{\delta}$ dense subset $E \subset \mathbb{T}$ such that the set of continuous functions whose Fourier expansion diverges on this set is a $G_{\delta}$ dense subset of $\mathcal{C}(\mathbb{T})([12$, p. 102]). While writing this paper, the authors became aware of recent work by F. Bayart ([4]) where using different techniques it is shown that, if $\mathcal{F}_{E} \subset \mathcal{C}(\mathbb{T})$ is the set of continuous functions whose Fourier series expansion diverges on a set of measure zero, $E$, then $\mathcal{F}_{E}$ is dense-lineable. This result is a consequence of our main result, Theorem 2.1.

Let us recall the following definition, introduced in [3]:

Definition. Given a Banach algebra $\mathcal{A}$ and a subset $\mathcal{B} \subset \mathcal{A}$, we say that:

(1) $\mathcal{B}$ is algebrable if there is a subalgebra $\mathcal{C}$ of $\mathcal{A}$ so that $\mathcal{C} \subset \mathcal{B} \cup\{0\}$ and the cardinality of any system of generators of $\mathcal{C}$ is infinite;

(2) $\mathcal{B}$ is dense-algebrable if, in addition, $\mathcal{C}$ can be taken dense in $\mathcal{A}$.

Recently ([3]) it was shown that the set of complex-valued everywhere surjective functions is algebrable. The aim of this paper is to show that, given any set $E \subset \mathbb{T}$ of measure zero, the set of continuous functions whose Fourier series expansion is divergent at any point $t \in E$ is dense-algebrable.

Here, and from now on, $\mathbb{T}$ denotes $\mathbb{R} / 2 \pi \mathbb{Z}, \widehat{f}(n)$ or $f^{\wedge}(n)$ will denote the $n$th Fourier coefficient of $f \in \mathcal{C}(\mathbb{T}), \mathcal{Z}(f)$ denotes the set $\{n \in \mathbb{Z}: \widehat{f}(n) \neq 0\}$, and $S_{n}(f, t)$ will denote the $n$th partial Fourier sum $\sum_{u=-n}^{n} \widehat{f}(u) e^{i u t}$.

\section{The main result}

TheOREM 2.1. Let $E \subset \mathbb{T}$ be a set of measure zero. Let $\mathcal{F}_{E} \subset \mathcal{C}(\mathbb{T})$ be the set of continuous functions whose Fourier series expansion diverges at every point $t \in E$. Then $\mathcal{F}_{E}$ is dense-algebrable. 
In order to prove this result, we need several different tools, amongst them some technical lemmas and the construction of a double sequence.

2.1. Preliminary elements. By $[9$, pp. 57-58], given a set $E \subset \mathbb{T}$ of measure zero we can find a sequence $\left(\widetilde{H}_{k}\right)_{k}$ of trigonometric polynomials, a sequence $\left(n_{k}\right)_{k}$ of positive integers, and a sequence $\left(E_{k}\right)_{k}$ of measurable subsets of $\mathbb{T}$ such that every $t \in E$ belongs to infinitely many $E_{k}$ 's and:

1. $\left|\widetilde{H}_{k}(s)\right| \leq 1$ for all $s \in \mathbb{T}$,

2. $\left|S_{n_{k}}\left(\widetilde{H}_{k}, t\right)\right| \geq 2^{k^{2}}$ for every $t \in E_{k}$.

Without loss of generality we may suppose that

$$
\widetilde{H}_{k}(t)=\sum_{r=0}^{\widetilde{a}_{k}} \widehat{\widetilde{H}}_{k}(r) e^{i r t},
$$

that is, $\mathcal{Z}\left(\widetilde{H}_{k}\right) \subset\left[0, \widetilde{a}_{k}\right]$, with $\left(\widetilde{a}_{k}\right)$ increasing, $\widetilde{a}_{k}>n_{k}$ for every $k \in \mathbb{N}$. Let us define $H_{k}(t)=1+e^{i\left(\widetilde{a}_{k}+1\right) t} \widetilde{H}_{k}(t)$ and let us call $a_{k}=2 \widetilde{a}_{k}+1$ (to have $\left.\mathcal{Z}\left(H_{k}\right) \subset\left[0, a_{k}\right]\right)$.

Let $\left(Q_{j}\right)_{j}$ be a sequence of trigonometric polynomials that is dense in $\mathcal{C}(\mathbb{T})$ and such that $\mathcal{Z}\left(Q_{j}\right) \subset\left[-q_{j}, q_{j}\right]$. We may clearly suppose that the sequence $\left(q_{j}\right)_{j}$ is increasing, and we let $b_{j}=\max \left\{a_{j}, q_{j}\right\}$.

Next, consider the following linear order $\prec$ on $\mathbb{N} \times \mathbb{N}$ :

$$
(j, k) \prec\left(j^{\prime}, k^{\prime}\right) \Leftrightarrow \begin{cases}j+k<j^{\prime}+k^{\prime} & \text { or } \\ j+k=j^{\prime}+k^{\prime} & \text { and } k<k^{\prime}\end{cases}
$$

This order can be represented by the following diagram, in which each arrow connects a pair with its immediate successor:

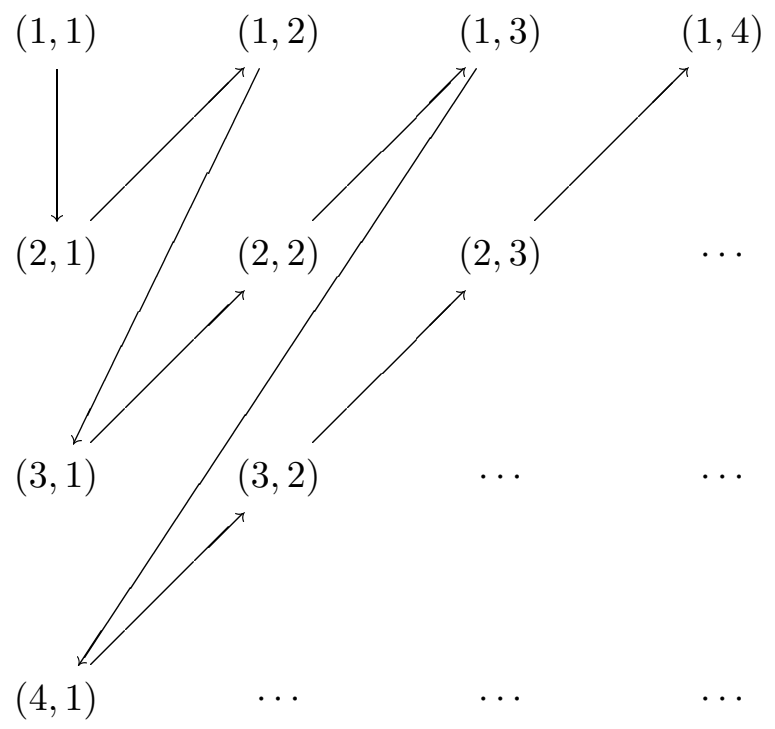


With this order we define by recursion the following double sequence $\left(p_{k}^{j}\right)_{j, k}$ :

$$
p_{1}^{1}=3 b_{1}+1, \quad p_{k}^{j}=3 k b_{k}+2 \max \left\{k, k^{\prime}\right\} p_{k^{\prime}}^{j^{\prime}}+1,
$$

where $\left(j^{\prime}, k^{\prime}\right)$ is the immediate predecessor of $(j, k)$.

Of course, $p_{k}^{j}<p_{k^{\prime}}^{j^{\prime}}$ if and only if $(j, k) \prec\left(j^{\prime}, k^{\prime}\right)$. Moreover $\left(p_{k}^{j}\right)_{j, k}$ is increasing in both indices and has, trivially, the following properties:

(P1) If $(j, k) \preceq\left(j^{\prime}, k^{\prime}\right)$ then $b_{k}<p_{k^{\prime}}^{j^{\prime}}$.

(P2) $p_{d}^{i}>3 d b_{d}$ for every $i, d$.

(P3) If $(j, k) \prec(i, d)$, then $p_{d}^{i}>2 d p_{k}^{j}+3 d b_{d}$.

(P4) If $(i, d) \prec(j, k)$, then $p_{k}^{j}>2 d p_{d}^{i}$.

2.2. The main construction. We now use the above elements to state and prove a technical lemma that will be necessary for the proof of the main result:

Lemma 2.2. Suppose that

$$
p_{d}^{i_{1}}+\cdots+p_{d}^{i_{n}}+u=p_{k_{1}}^{j_{1}}+\cdots+p_{k_{s}}^{j_{s}}+v
$$

and:

(i) $s \leq n \leq d$;

(ii) $p_{d}^{i_{1}} \geq p_{d}^{i_{2}} \geq \cdots \geq p_{d}^{i_{n}}$ (that is, $i_{1} \geq \cdots \geq i_{n}$ );

(iii) $p_{k_{1}}^{j_{1}} \geq p_{k_{2}}^{j_{2}} \geq \cdots \geq p_{k_{s}}^{j_{s}}$;

(iv) $|u| \leq d b_{d}$;

(v) $-d b_{d} \leq v \leq b_{k_{1}}+\cdots+b_{k_{s}}+d b_{d}$.

Then $n=s, i_{r}=j_{r}, k_{r}=d$ for every $r$ and $u=v$.

Proof. If $p_{d}^{i_{1}}>p_{k_{1}}^{j_{1}}$, then

$$
\begin{aligned}
& p_{d}^{i_{1}} \stackrel{\text { (P3) }}{>} 2 d p_{k_{1}}^{j_{1}}+3 d b_{d} \stackrel{\text { (i) }}{\geq} 2 s p_{k_{1}}^{j_{1}}+3 d b_{d} \\
& \stackrel{(\mathrm{P} 1)+(\mathrm{iii})}{>} p_{k_{1}}^{j_{1}}+\cdots+p_{k_{s}}^{j_{s}}+b_{k_{1}}+\cdots+b_{k_{s}}+3 d b_{d}
\end{aligned}
$$

and, by (iv) and (v), we cannot have equation (1).

If $p_{k_{1}}^{j_{1}}>p_{d}^{i_{1}}$, then

$$
p_{k_{1}}^{j_{1}} \stackrel{(\mathrm{P} 4)}{>} 2 d p_{d}^{i_{1}} \stackrel{(\mathrm{P} 2)}{>} d p_{d}^{i_{1}}+3 d b_{d} \stackrel{(\mathrm{i})}{\geq} n p_{d}^{i_{1}}+3 d b_{d} \stackrel{\text { (ii) }}{\geq} p_{d}^{i_{1}}+\cdots+p_{d}^{i_{n}}+3 d b_{d}
$$

and, by (iv) and (v), we cannot have equation (1).

So $i_{1}=j_{1}, k_{1}=d$ and we can convert (1) into

$$
p_{d}^{i_{2}}+\cdots+p_{d}^{i_{n}}+u=p_{k_{2}}^{j_{2}}+\cdots+p_{k_{s}}^{j_{s}}+v
$$

where $-d b_{d} \leq v \leq b_{k_{2}}+\cdots+b_{k_{s}}+(d+1) b_{d}$. 
Reasoning in the same way we can obtain $i_{2}=j_{2}$ and $k_{2}=d$, reducing equation (2) to

$$
p_{d}^{i_{3}}+\cdots+p_{d}^{i_{n}}+u=p_{k_{3}}^{j_{3}}+\cdots+p_{k_{s}}^{j_{s}}+v
$$

where $-d b_{d} \leq v \leq b_{k_{3}}+\cdots+b_{k_{s}}+(d+2) b_{d}$.

Continuing with this procedure (recall that, by (i), $s \leq n$ ) we arrive at

$$
p_{d}^{i_{s}}+\cdots+p_{d}^{i_{n}}+u=p_{k_{s}}^{j_{s}}+v
$$

where $-d b_{d} \leq v \leq b_{k_{s}}+(d+s-1) b_{d}$. With the same arguments one can conclude that $j_{s}=i_{s}$ and $k_{s}=d$. So it remains to prove that $n=s$. If not, then $n \geq s+1$, and from (1), we obtain $v=p_{d}^{i_{s+1}}+\cdots+p_{d}^{i_{n}}+u$ with $|u| \leq d b_{d}$ and $-d b_{d} \leq v \leq(d+s) b_{d} \leq 2 d b_{d}$. Now

$$
v=p_{d}^{i_{s+1}}+\cdots+p_{d}^{i_{n}}+u \geq p_{d}^{i_{s+1}}+u \stackrel{(\mathrm{P} 2)}{>} 3 d b_{d}-d b_{d}=2 d b_{d} \geq v,
$$

and we reach a contradiction.

Next, for each $j \geq 1$ and $m \geq 1$, let us define the function

$$
f_{j}^{m}(t)=\sum_{k=1}^{m} 2^{-k} \cdot e^{i p_{k}^{j} t} H_{k}(t) .
$$

Thanks to the Weierstrass $M$-test we know that, for each $j \in \mathbb{N}$, the sequence $\left(f_{j}^{m}\right)_{m}$ converges uniformly to a continuous function $f_{j} \in \mathcal{C}(\mathbb{T})$ with $\left\|f_{j}\right\| \leq 2$. Define $g_{j}=(1 / j) f_{j}+Q_{j}$ and let $\mathcal{A}$ be the algebra generated by $\left\{g_{j}\right\}_{j}$.

Since $\left(Q_{j}\right)_{j}$ is a dense sequence in $\mathcal{C}(\mathbb{T})$, it is clear that $\left(g_{j}\right)_{j}$ (and hence $\mathcal{A})$ is also dense in $\mathcal{C}(\mathbb{T})$. This immediately implies that $\mathcal{A}$ is infinitedimensional.

We will see that every $g \in \mathcal{A} \backslash\{0\}$ has a Fourier series divergent at any $t \in E$. So let us take a generic element of $\mathcal{A}$ :

$$
g=\sum_{j=1}^{N} \alpha_{j} g_{i_{1}^{j}} \cdots g_{i_{s_{j}}^{j}}
$$

where $s_{1} \geq \cdots \geq s_{N}$ ( $s_{j}$ is the number of functions that we multiply in each summand of $g$ ) and $i_{1}^{j} \geq \cdots \geq i_{s_{j}}^{j}$ for every $j$.

The heart of the matter is the next technical lemma:

LEMma 2.3. If $d \geq \max \left\{s_{1}, i_{1}^{1}, \ldots, i_{1}^{N}\right\}$ then:

(a) $\widehat{g}\left(p_{d}^{i_{1}^{1}}+\cdots+p_{d}^{i_{s_{1}}^{1}}+u\right)=\alpha_{1}\left(i_{1}^{1} \cdots i_{s_{1}}^{1}\right)^{-1} 2^{-s_{1} d} \widehat{H_{d}^{s_{1}}}(u)$ for $0 \leq u \leq s_{1} a_{d}$.

(b) $\widehat{g}(u)=0$ for $u \leq-\left(p_{d}^{i_{1}^{1}}+\cdots+p_{d}^{i_{s_{1}}^{1}}\right)$.

(c) If $l>i_{1}^{1}$, then $\widehat{g}_{l}\left(p_{d}^{i_{1}^{1}}+\cdots+p_{d}^{i_{s_{1}}^{1}}+u\right)=0$ for every $0 \leq u \leq s_{1} a_{d}$ and hence $g_{l} \neq g$. 
Proof. First of all, notice that if we expand the sum

$$
g=\sum_{j=1}^{N} \alpha_{j}\left(Q_{i_{1}^{j}}+\frac{1}{i_{1}^{j}} f_{i_{1}^{j}}\right) \cdots\left(Q_{i_{s_{j}}^{j}}+\frac{1}{i_{s_{j}}^{j}} f_{i_{s_{j}}^{j}}\right),
$$

we obtain terms of the form $f_{h_{1}} \cdots f_{h_{r}} Q_{h_{r+1}} \cdots Q_{h_{s}}$ with $s \leq s_{1} \leq d$ and $d \geq h_{j}$ for every $1 \leq j \leq s$ (we can suppose that $h_{1} \geq \cdots \geq h_{r}$ ).

By the uniform convergence of the sequence $\left(f_{j}^{m}\right)_{m}$, the sequence $\left(f_{h_{1}}^{m} \cdots f_{h_{r}}^{m} Q_{h_{r+1}} \cdots Q_{h_{s}}\right) \uparrow(v)$ converges to $\left(f_{h_{1}} \cdots f_{h_{r}} Q_{h_{r+1}} \cdots Q_{h_{s}}\right)^{\wedge}(v)$ for every $v$. It is easy to see that this sequence is in fact constant from some $m$ on. Now, for a fixed $m$, the function $f_{h_{1}}^{m} \cdots f_{h_{r}}^{m} Q_{h_{r+1}} \cdots Q_{h_{s}}$ is a trigonometric polynomial whose non-zero Fourier coefficients are contained in the set

$$
\begin{aligned}
A^{m}:= & \left\{p_{k_{1}}^{h_{1}}+\cdots+p_{k_{r}}^{h_{r}}+v:\right. \\
& -q_{h_{r+1}}-\cdots-q_{h_{s}} \leq v \leq a_{k_{1}}+\cdots+a_{k_{r}}+q_{h_{r+1}}+\cdots+q_{h_{s}}, \\
1 & \left.\leq k_{1}, \ldots, k_{r} \leq m\right\} .
\end{aligned}
$$

Therefore, the non-zero Fourier coefficients of $f_{h_{1}} \cdots f_{h_{r}} Q_{h_{r+1}} \cdots Q_{h_{s}}$ are contained in $A=\bigcup_{m} A^{m}$ and hence in $B:=\left\{p_{k_{1}}^{h_{1}}+\cdots+p_{k_{r}}^{h_{r}}+v:-d b_{d} \leq v \leq b_{k_{1}}+\cdots+b_{k_{r}}+d b_{d}, k_{1}, \ldots, k_{r} \geq 1\right\}$.

Since $B \subset\left[-d b_{d},+\infty\right)$, (P2) gives (b).

Now, Lemma 2.2 tells us that, if $p_{d}^{i_{1}^{1}}+\cdots+p_{d}^{i_{s_{1}}^{1}}+u\left(|u| \leq d b_{d}\right)$ belongs to $B$, then $r=s_{1}, h_{1}=i_{1}^{1}, \ldots, h_{r}=i_{s_{1}}^{1}$ and $k_{1}=\cdots=k_{r}=d$. Thus,

$$
\widehat{g}\left(p_{d}^{i_{1}^{1}}+\cdots+p_{d}^{i_{s_{1}}^{1}}+u\right)=\alpha_{1} \frac{1}{i_{1}^{1} \cdots i_{s_{1}}^{1}}\left(f_{i_{1}^{1}} \cdots f_{i_{s_{1}}^{1}}\right)^{\wedge}\left(p_{d}^{i_{1}^{1}}+\cdots+p_{d}^{i_{s_{1}}^{1}}+u\right),
$$

for $|u| \leq d b_{d}$.

Moreover, Lemma 2.2 also says that $p_{d}^{i_{1}^{1}}+\cdots+p_{d}^{i_{s_{1}}^{1}}+u\left(|u| \leq d b_{d}\right)$ is different from any other $p_{k_{1}}^{i_{1}^{1}}+\cdots+p_{k_{s_{1}}}^{i_{s_{1}}^{1}}+v$ with $-d b_{d} \leq v \leq b_{k_{1}}+\cdots+$ $b_{k_{r}}+d b_{d}$.

Thus, looking at the formula

$$
\begin{aligned}
& f_{i_{1}^{1}}^{m} \cdots f_{i_{s_{1}}^{1}}^{m}(t) \\
& =\sum_{k_{1}, \ldots, k_{s_{1}}=1}^{m} \sum_{r_{1}, \ldots, r_{s_{1}}=0}^{a_{k_{1}}, \ldots, a_{k_{s_{1}}}} 2^{-k_{1}-\cdots-k_{s_{1}}} \widehat{H}_{k_{1}}\left(r_{1}\right) \cdots \widehat{H}_{k_{s_{1}}}\left(r_{s_{1}}\right) e^{i\left(p_{k_{1}}^{i_{1}^{1}}+\cdots+p_{k_{s_{1}}}^{i_{s_{1}}^{1}}+r_{1}+\cdots+r_{s_{1}}\right) t}
\end{aligned}
$$

one finds that, if $0 \leq u \leq s_{1} a_{d}$, then

$$
\left(f_{i_{1}^{1}}^{m} \cdots f_{i_{s_{1}}^{1}}^{m}\right)^{\wedge}\left(p_{d}^{i_{1}^{1}}+\cdots+p_{d}^{i_{s_{1}}^{1}}+u\right)=\sum_{\substack{r_{1}+\cdots+r_{s_{1}}=u \\ 0 \leq r_{1}, \ldots, r_{s_{1}} \leq a_{d}}} 2^{-s_{1} d} \widehat{H_{d}}\left(r_{1}\right) \cdots \widehat{H_{d}}\left(r_{s_{1}}\right),
$$

which is exactly $2^{-s_{1} d} \widehat{H_{d}^{s_{1}}}(u)$, and this gives (a). 
Finally, to obtain (c) we can reason in the same way. The non-zero Fourier coefficients of $g_{l}$ are in the set $C=\left\{p_{k}^{l}+v: 0 \leq v \leq b_{k}\right\}$. Now, thanks to Lemma 2.2 , if $p_{k}^{l}+v=p_{d}^{i_{1}^{1}}+\cdots+p_{d}^{i_{s_{1}}^{1}}+u$ for some $0 \leq v \leq b_{k}$, $0 \leq u \leq s_{1} a_{d} \leq d b_{d}$, we obtain $i_{1}^{1}=l$, which is impossible since $l>i_{1}^{1}$. This completes the proof of the lemma.

Proof of Theorem 2.1. Now, set $\beta_{1}=\alpha_{1}\left(i_{1}^{1} \cdots i_{s_{1}}^{1}\right)^{-1}$ and take any $d \geq$ $\max \left\{s_{1}, i_{1}^{1}, \ldots, i_{1}^{N}\right\}$. It follows that

$$
\begin{aligned}
& \left|S_{p_{d}^{1}+\cdots+p_{d}^{1}+i_{1}^{1}+\widetilde{a}_{d}+1+n_{d}}(g, t)-S_{p_{d}^{1}+\cdots+p_{d}^{1 \frac{1}{1}}+\widetilde{a}_{d}}(g, t)\right| \\
& =\left|\sum_{-\left(p_{d}^{i_{1}^{1}}+\cdots+p_{d}^{i_{s_{1}}}+\widetilde{a}_{d}+1+n_{d}\right)}^{-\left(p_{d}^{i_{1}^{1}}+\cdots+p_{d}^{i_{s_{1}}^{1}}+\widetilde{a}_{d}+1\right)} \widehat{g}(u) e^{i u t}+\sum_{p_{d}^{i_{1}^{1}}+\cdots+p_{d}^{i_{1}^{1}}+\widetilde{a}_{d}+1}^{p_{d}^{i_{1}^{1}}+\cdots+p_{d}^{i_{s_{1}}^{1}}+\widetilde{a}_{d}+1+n_{d}} \widehat{g}(u) e^{i u t}\right| .
\end{aligned}
$$

By Lemma 2.3(b), the first term of (3) is zero. Thus, by Lemma 2.3(a), (3) is equal to

$$
\beta_{1} 2^{-s_{1} d}\left|\sum_{u=\widetilde{a}_{d}+1}^{\widetilde{a}_{d}+1+n_{d}} \widehat{H_{d}^{s_{1}}}(u) e^{i u t}\right|
$$

But, by the definition of $H_{d}$, we have

$$
H_{d}^{s_{1}}(t)=\sum_{k=0}^{s_{1}}\left(\begin{array}{c}
s_{1} \\
k
\end{array}\right) e^{i k\left(\widetilde{a}_{d}+1\right) t} \widetilde{H}_{d}^{k}(t)
$$

and, since $\mathcal{Z}\left(\widetilde{H}_{d}^{k}\right) \subset[0, \infty)$ for any $k$, we see that for $u \in\left[\widetilde{a}_{d}+1, \widetilde{a}_{d}+1+n_{d}\right]$,

$$
\widehat{H_{d}^{s_{1}}}(u)=s_{1} \widehat{\widetilde{H}}_{d}\left(u-\left(\widetilde{a}_{d}+1\right)\right) \quad\left(\text { recall that } n_{d} \leq \widetilde{a}_{d}\right) .
$$

Therefore (3) equals

$$
\beta_{1} s_{1} 2^{-s_{1} d}\left|\sum_{u=0}^{n_{d}} \widehat{\widetilde{H}}_{d}(u) e^{i u t}\right|=\beta_{1} s_{1} 2^{-s_{1} d}\left|S_{n_{d}}\left(\widetilde{H}_{d}, t\right)\right| \geq \beta_{1} s_{1} 2^{d^{2}-s_{1} d}
$$

for every $t \in E_{d}$. Since any $t \in E$ belongs to infinitely many $E_{d}$ 's, we have

$$
\limsup _{n}\left|S_{n}(g, t)\right|=\infty \quad \text { for every } t \in E .
$$

To finish the proof of Theorem 2.1 it only remains to prove that $\mathcal{A}$ is infinitely generated. Now, if $\mathcal{A}$ were finitely generated, there would exist an $l \in \mathbb{N}$ such that every $g_{h}, h \in \mathbb{N}$, can be expressed as

$$
g_{h}=\sum_{j=1}^{N} \alpha_{j} g_{i_{1}^{j}} \cdots g_{i_{s_{j}}^{j}}
$$

with $s_{1} \geq \cdots \geq s_{N}$ and $l>i_{1}^{j} \geq \cdots \geq i_{s_{j}}^{j}$ for every $j$. Taking $h=l$ in (4) this contradicts Lemma 2.3(c), which finishes the proof of Theorem 2.1. 


\section{References}

[1] R. M. Aron, D. García and M. Maestre, Linearity in non-linear problems, Rev. R. Acad. Cienc. Exactas Fís. Nat. Ser. A Mat. 95 (2001), 7-12.

[2] R. M. Aron, V. I. Gurariy and J. B. Seoane-Sepúlveda, Lineability and spaceability of sets of functions on $\mathbb{R}$, Proc. Amer. Math. Soc. 133 (2005), 795-803.

[3] R. M. Aron and J. B. Seoane-Sepúlveda, Algebrability of the set of everywhere surjective functions on $\mathbb{C}$, Bull. Belg. Math. Soc. Simon Stevin, to appear.

[4] F. Bayart, Topological and algebraic genericity of divergence and universality, Studia Math. 167 (2005), 153-160.

[5] P. Enflo and V. Gurariy, On lineability and spaceability of sets in function spaces, preprint.

[6] V. P. Fonf, V. I. Gurariy and M. I. Kadets, An infinite-dimensional subspace of $\mathcal{C}[0,1]$ consisting of nowhere differentiable functions, C. R. Acad. Bulgare Sci. 52 (1999), no. 11-12, 13-16.

[7] V. I. Gurariy and L. Quarta, On lineability of sets of continuous functions, J. Math. Anal. Appl. 294 (2004), 62-72.

[8] S. Hencl, Isometrical embeddings of separable Banach spaces into the set of nowhere approximatively differentiable and nowhere Hölder functions, Proc. Amer. Math. Soc. 128 (2000), 3505-3511.

[9] V. Katznelson, An Introduction to Harmonic Analysis, Dover, 1968.

[10] T. W. Körner, Fourier Analysis, Cambridge Univ. Press, 1988.

[11] L. Rodríguez-Piazza, Every separable Banach space is isometric to a space of continuous nowhere differentiable functions, Proc. Amer. Math. Soc. 123 (1995), 36493654 .

[12] W. Rudin, Real and Complex Analysis, 3rd ed., McGraw-Hill, New York, 1987.

Department of Mathematics

Kent State University

Kent, OH 44242, U.S.A.

E-mail: aron@math.kent.edu jseoane@math.kent.edu
Departamento de Matemática Aplicada E.S.C.E.T. - Universidad Rey Juan Carlos 28933 Móstoles, Madrid, Spain E-mail: david.perez.garcia@urjc.es

Received September 9, 2005

Revised version February 10, 2006 